University of Nebraska - Lincoln

DigitalCommons@University of Nebraska - Lincoln

2010

\title{
Interaction of Bacillus species and Salmonella enterica serovar Typhimurium in immune or inflammatory signaling from swine intestinal epithelial cells
}

\author{
C. C. Aperce \\ Kansas State University \\ Thomas E. Burkey \\ University of Nebraska-Lincoln, tburkey2@unl.edu \\ B. KuKanich \\ Kansas State University \\ B. A. Crozier-Dodson \\ Kansas State University \\ S. S. Dritz \\ Kansas State University \\ See next page for additional authors \\ Follow this and additional works at: https://digitalcommons.unl.edu/animalscifacpub \\ Part of the Animal Sciences Commons
}

Aperce, C. C.; Burkey, Thomas E.; KuKanich, B.; Crozier-Dodson, B. A.; Dritz, S. S.; and Minton, J. E., "Interaction of Bacillus species and Salmonella enterica serovar Typhimurium in immune or inflammatory signaling from swine intestinal epithelial cells" (2010). Faculty Papers and Publications in Animal Science. 700.

https://digitalcommons.unl.edu/animalscifacpub/700

This Article is brought to you for free and open access by the Animal Science Department at DigitalCommons@University of Nebraska - Lincoln. It has been accepted for inclusion in Faculty Papers and Publications in Animal Science by an authorized administrator of DigitalCommons@University of Nebraska - Lincoln. 


\section{Authors}

C. C. Aperce, Thomas E. Burkey, B. KuKanich, B. A. Crozier-Dodson, S. S. Dritz, and J. E. Minton 


\title{
Interaction of Bacillus species and Salmonella enterica serovar Typhimurium in immune or inflammatory signaling from swine intestinal epithelial cells ${ }^{1}$
}

\author{
C. C. Aperce, ${ }^{*}$ T. E. Burkey,† B. KuKanich, + B. A. Crozier-Dodson, ${ }^{*}$ S. S. Dritz, $\S$ \\ and J. E. Minton*2 \\ *Department of Animal Sciences and Industry, Kansas State University, Manhattan 66506-0201; \\ $\dagger$ Department of Animal Sciences, University of Nebraska, Lincoln 68583-0908; \\ $\ddagger$ Department of Anatomy and Physiology, Kansas State University, Manhattan 66506-5802; \\ and $\S$ Department of Diagnostic Medicine/Pathobiology, Kansas State University, Manhattan 66506-5601
}

\begin{abstract}
Previous research evaluated a laboratory strain of Bacillus licheniformis (BL) in a model swine epithelium and found it exerted antiinflammatory effects on Salmonella enterica serovar Typhimurium (Sal)-induced secretion of IL-8. The current investigation evaluated the antiinflammatory actions of Bacillus bacteria available commercially as feed additives for the swine industry. Three isolates were obtained from the product, 2 Bacillus subtilis (BS1 and BS3) and 1 BL (BL2). Swine jejunal epithelial IPEC-J2 cells were seeded into wells on permeable membrane supports and allowed to form confluent monolayers. Treatments included apical pretreatment with BL, BS1, BL2, or BS3 for $17 \mathrm{~h}$ without Sal, and the same Bacillus treatments but with $10^{8} \mathrm{cfu}$ of Sal added in the final hour of $\mathrm{Ba}$ cillus incubation. Two additional treatments included negative control wells receiving no bacteria (control) and positive control wells receiving only Sal (10 total treatments). After bacterial incubation, wells were washed and fresh medium containing gentamicin was added. Cells were incubated for an additional $5 \mathrm{~h}$, after which apical and basolateral media were recovered
\end{abstract}

for determination of IL-8 and bacitracin. In addition, inserts with epithelial cells that had received Sal were lysed and lysates were cultured to determine treatment effects on Sal invasion. Exposure to Sal alone provoked an increase in IL-8 secretion from IPEC-J2 cells compared with control wells $(P<0.001$ for both the apical and basolateral directions). Pretreatment with each Bacillus isolate followed by challenge with Sal reduced Sal-induced IL-8 secretion in both the apical and basolateral compartments compared with wells receiving only Sal $(P<0.001$; except for BS3 apical, $P<0.01)$. The residual presence of bacitracin could be detected only in BL2 and BL2+Sal. Fewer Sal colonies could be cultured from lysates of BL2+Sal than from the Sal, $\mathrm{BS} 1+\mathrm{Sal}$, and $\mathrm{BS} 3+\mathrm{Sal}$ treatments $(P<0.001)$. Results indicate that $B$. subtilis and BL have the ability to intervene in secretion of the neutrophil chemoattractant IL-8 from swine intestinal epithelial cells. This effect on chemokine secretion by gastrointestinal epithelial cells in vitro could not be explained solely by reduced invasion of epithelial cells by Sal.

Key words: Bacillus, intestinal epithelium, Salmonella, swine

(C)2010 American Society of Animal Science. All rights reserved.

J. Anim. Sci. 2010. 88:1649-1656

doi:10.2527/jas.2009-2263

\section{INTRODUCTION}

The growth response of nursery pigs to in-feed antibiotics is well documented (Dritz et al., 2002). To date, no single additive or class of additives has been identi-

\footnotetext{
${ }^{1}$ Contribution no. 09-388-J from the Kansas Agricultural Experiment Station (Manhattan). The authors thank C. M. Hill (Department of Animal Sciences and Industry, Kansas State University, Manhatten) for technical assistance in the laboratory.

${ }^{2}$ Corresponding author: eminton@ksu.edu

Received July 1, 2009.

Accepted December 30, 2009.
}

fied to replace the growth response of nursery pigs to in-feed antibiotics. However, the search for nonantibiotic replacements continues, with the direct-fed microbials representing a significant portion of that search. In general, evaluation of direct-fed microbials, at times referred to as probiotics, has been largely empirical. Little is actually known to suggest how direct-fed microbials may interact with enterocytes in the presence of pathogenic organisms that are presumably controlled (to some extent) by growth-promoting quantities of dietary antibiotics. Bacillus spp. represent a collection of species that may have potential as direct-fed micro- 
bials. Bacillus bacteria are attractive because of their well-established ability to sporulate and their tendency to produce secondary metabolites (US Environmental Protection Agency, 1997a,b). To that end, our laboratory previously evaluated a laboratory strain of $B a$ cillus licheniformis (BL) in a model swine epithelium and found it to intervene significantly in Salmonella enterica serovar Typhimurium (Sal)-induced secretion of IL-8 from gut epithelial cells (Skjolaas et al., 2007). Additional preliminary results suggested that the antiinflammatory effects of BL were time dependent (Godsey et al., 2007). The current investigation was undertaken to further evaluate the antiinflammatory actions of Bacillus spp. in a model swine gut epithelium. We specifically sought to evaluate these effects using Bacillus bacteria available commercially as direct-fed microbial feed additives for the swine industry.

\section{MATERIALS AND METHODS}

Animal Care and Use Committee approval was not obtained for this study because no animals were used.

The aim of this study was to investigate the interaction of Bacillus bacteria and Sal in immune or inflammatory signaling from swine intestinal epithelial cells. Our previous investigation was limited to a laboratory strain of BL (Skjolaas et al., 2007). To gain access to Bacillus bacteria of relevance to the swine industry, we isolated strains from a commercial product (BioPlus 2B, Chr. Hansen, Milwaukee, WI) for the current studies.

\section{Bacterial Isolation}

A sample of the commercial feed additive $(25 \mathrm{~g})$ was solubilized in $225 \mathrm{~mL}$ of sterile water and mixed. A 1-mL quantity of the solution was then diluted in $9 \mathrm{~mL}$ of trypticase soy broth (TSB; MP Biomedicals LLC, Solon, OH). After an overnight incubation at $37^{\circ} \mathrm{C}$, a trypticase soy agar (TSA; MP Biomedicals) plate was prepared using the broth and incubated overnight. Three different types of colonies were isolated. Colonies were forwarded to a commercial laboratory for identification (Silliker Inc., St. Louis, MO). Specimens 1 and 3 were identified as Bacillus subtilis (hereafter, BS1 and BS3, respectively). Specimen 2 was identified as BL (hereafter, BL2).

The Sal and the BL isolates used for additional treatments were the same isolates used previously in our laboratory (Skjolaas et al., 2007). In brief, the Sal was isolated from a clinical case of swine enteric disease and the BL isolate was a laboratory strain obtained commercially [American Type Culture Collection (ATCC), Manassas, VA].

\section{Growth Curves}

The growth behavior of bacterial isolates was important in the design and execution of the experiment, particularly the growth of sufficient numbers of Bacillus bacteria to distribute among treatments. Growth curves were established for each bacterial isolate in TSB. For this purpose, the absorbance of the broth at $600 \mathrm{~nm}$ was measured, followed by a standard bacterial plate count. After an overnight incubation at $37^{\circ} \mathrm{C}$, colonies were counted and the bacterial population was estimated.

\section{Bacterial Sensitivity}

The sensitivity of the bacteria to common antibiotics was assessed using a microplate assay. Bacteria were cultured on TSA and incubated overnight at $37^{\circ} \mathrm{C}$. Three to 5 colonies were then picked and placed in distilled water to obtain a turbidity of 0.5 McFarland turbidity standard $\left(10^{8} \mathrm{cfu}\right)$. A $100-\mu \mathrm{L}$ quantity of the suspension was added to a 9 -mL tube of Mueller-Hinton broth, and $50 \mu \mathrm{L}$ of the final solution was added in each well of the microplate containing antibiotics at various dilutions. The plates were incubated for 24 $\mathrm{h}$ at $37^{\circ} \mathrm{C}$. The smallest antibiotic concentration that completely inhibited visible growth was considered to be the minimum inhibitory concentration. Qualitative data concerning antibiotic sensitivity were used to ensure bacteria cultured out of epithelial cells were Sal rather than one of the Bacillus spp.

\section{Culture of Epithelial Cells}

The swine jejunal epithelial cell line, IPEC-J2, was used to assess the interaction of Sal and the various $B a$ cillus isolates (Rhoads et al., 1994). Culture conditions were identical to those described previously (Skjolaas et al., 2006, 2007), except that IPEC-J2 cells (passages 61 to 70) were cultured in 24-mm, 6-well Costar Snapwells (Corning Inc., Corning, NY) for exactly $7 \mathrm{~d}$ before executing the experiment.

\section{Exposure of IPEC-J2 Cells to Bacteria}

Twenty-four hours before the beginning of the experiment, confluent IPEC-J2 cells were washed twice with PBS and fresh antibiotic-free medium was added. Bacillus bacteria were grown on TSB to obtain the required concentration.

Design of the bacterial exposure of epithelial cells was patterned after our previously published study (Skjolaas et al., 2007). There were 10 treatments, and this required each replicate of the experiment to occupy 2 culture plates. Eight of the 10 treatments required preexposure of IPEC-J2 in the apical chamber to a 17 -h incubation with Bacillus isolates $\left(10^{8} \mathrm{cfu} /\right.$ well). There were 4 Bacillus isolates, 3 of which were from the commercial product (BS1, BL2, and BS3) and 1 of which was the ATCC strain (BL) used previously (Skjolaas et al., 2007). After $16 \mathrm{~h}$ had elapsed, one-half the wells containing Bacillus bacteria were treated apically with $10^{8} \mathrm{cfu}$ of Sal and the other one-half received 
a similar volume of sterile culture media. To recap, the 8 treatment combinations were BL, BS1, BL2, and BS3 without and with Sal coculture (BL+Sal, BS1+Sal, BL2+Sal, and BS3+Sal, respectively). The other 2 (of 10 total) treatments were negative control wells receiving no bacteria (Con) and positive control wells receiving only Sal for $1 \mathrm{~h}$. Each run of the experiment was conducted on 4 separate dates. Within each run, there were 3 replicate wells for each treatment.

After the 1-h incubation after addition of Sal, all wells were washed twice by overflooding of PBS to remove the extracellular bacteria. New growth medium containing $50 \mu \mathrm{g} / \mathrm{mL}$ of gentamicin (Gibco, Grand Island, NY) was added to both the apical and basolateral wells. Plates were returned to the incubator for an additional $5 \mathrm{~h}$. Finally, the media from both apical and basolateral compartments were collected and stored for later IL-8 determination by ELISA as described previously (Skjolaas et al., 2006, 2007). An aliquot of the medium was also used to determine concentration of bacitracin (described in detail below).

\section{Sal Invasion into IPEC-J2 Epithelial Cells}

After removal of the media, inserts containing IPEC-J2 cells that had received treatment with Sal were washed twice with PBS, placed in new plates, and treated with $1 \mathrm{~mL}$ of $0.1 \%$ Triton $\mathrm{X}-100$. The Triton $\mathrm{X}-100$ solution was pipetted up and down to disrupt the epithelial cells thoroughly. Dilutions of the cell lysate were then applied to TSA plates that contained 250 $\mu \mathrm{g} / \mathrm{mL}$ of sulfadimethoxine. All Bacillus isolates had previously been determined to be sensitive to this antibiotic, whereas our Sal isolate was not. After an overnight incubation at $37^{\circ} \mathrm{C}$, colonies were counted and the number of colony-forming units of Sal per milliliter of IPEC-J2 lysate was determined.

\section{Bacitracin Assay}

Liquid chromatography coupled with electrosprayionization mass spectrometry (LCMS) was used to determine bacitracin production by the various Bacillus bacteria used in the experiment. Commercial bacitracin was purchased from Sigma-Aldrich (Vetranal, analytical standard, Riedel-deHaen, Sigma-Aldrich, St. Louis, MO). Bacitracin standard solutions were prepared by dilution of the commercial bacitracin in Dulbecco's modified Eagle's medium/F12 growth medium (with gentamicin) at the concentrations of 50, 100, 500, $1,000,5,000$, and 10,000 ng/mL. Samples (apical and basolateral media) were thawed and mixed thoroughly. Samples and standards were deproteinized by mixing $100 \mu \mathrm{L}$ of media with $200 \mu \mathrm{L}$ of methanol (100\%). The mixtures were again mixed well and centrifuged for 5 min at maximum speed $\left(16,100 \times g\right.$ at $\left.4^{\circ} \mathrm{C}\right)$. A $200-\mu \mathrm{L}$ quantity of the supernatant was then transferred to injection vials for LCMS analysis.
The assay was optimized for bacitracin A only considering that bacitracin $\mathrm{A}$ is the predominant form of bacitracin produced (Konz et al., 1997). Chromatographic separation was performed on a Supelco Discovery C8 column $(50 \times 2.1 \mathrm{~mm} \times 5 \mu M$; Sigma-Aldrich $)$. The mobile phase was a mixture of acetonitrile (A) and $0.1 \%$ formic acid $(\mathbf{B})$, and was delivered at a flow rate of $0.4 \mathrm{~mL} / \mathrm{min}$ under a gradient elution program (0 to 3 min, $5 \%$ A:95\% B; 3 to $5 \min , 30 \%$ A:70\% B; 5 to 6 $\min , 5 \% \mathrm{~A}: 95 \% \mathrm{~B} ; 6$ min to the end, $5 \% \mathrm{~A}: 95 \% \mathrm{~B})$ at room temperature. A delay was observed between each injection to restore the initial conditions. The qualifying and quantifying ion mass-to-charge ratios $(\mathrm{m} / \mathrm{z})$ used in the mass spectrometry interface were, respectively, 475.1 and 199.2. Settings, data acquisition, and processing were monitored by the software package Analyst version 1.5 (Applied Biosystems, Foster City, $\mathrm{CA}$ ). The time of retention of bacitracin was approximately $4 \mathrm{~min}$. The results were expressed as counts per second and were converted to nanograms of bacitracin per milliliter of media, and then further converted to nanograms per well.

\section{Sal Sensitivity to Bacitracin}

The sensitivity of Sal to bacitracin was established by a microdilution assay following the guidance of the Clinical and Laboratory Standards Institute (Wayne, PA). The commercial bacitracin was diluted 1:2 to create a dilution range from 39 to $20,000 \mathrm{ng} / \mathrm{mL}$ and 0.05 $\mathrm{mL}$ of each dilution was added into the wells of the microplate. Salmonella Typhimurium was cultured in Mueller Hinton cation-adjusted media (BD Diagnostic Systems, Franklin Lakes, NJ). The broth concentration was adjusted to $5 \times 10^{5} \mathrm{cfu} / \mathrm{mL}$ and $0.05 \mathrm{~mL}$ of the broth was added to the various concentrations of bacitracin in the microplate. Plates were incubated for $18 \mathrm{~h}$ and were then read at $590 \mathrm{~nm}$.

\section{Statistical Analyses}

Apical and basolateral concentrations of IL-8 were converted to nanograms per well to account for the difference in volume of the apical $(1.5 \mathrm{~mL})$ vs. the basolateral $(2.6 \mathrm{~mL})$ chamber. Concentrations of IL- 8 in the apical and basolateral compartments were analyzed using the MIXED procedure (SAS Inst. Inc., Cary, NC). The model included the fixed effects of treatment, secretion direction (apical or basolateral), and their interaction. Day was included in the model as a random effect. The MIXED procedure was also used to determine treatment effects for Sal invasion into IPEC-J2 epithelial cells. In this case, treatment was the sole source of variation in the model. To ensure normality of the data, raw colony-forming unit values were square root transformed. Means (and SEM) were back transformed for presentation of the data. The bacitracin data were analyzed using the MIXED procedure with treatment 
Table 1. Sample rRNA sequence comparison of specimens obtained from a commercial product with sequences in a reference library ${ }^{1}$

\begin{tabular}{|c|c|c|c|c|c|c|}
\hline \multirow[b]{2}{*}{ Library database } & \multicolumn{2}{|c|}{ Specimen 1} & \multicolumn{2}{|c|}{ Specimen 2} & \multicolumn{2}{|c|}{ Specimen 3} \\
\hline & Match, \% & Mismatches & Match, \% & Mismatches & Match, \% & Mismatches \\
\hline Bacillus subtilis subtilis (ATCC 6051) ${ }^{2}$ & 99.93 & 2 & 98.1 & 12 & 99.99 & 2 \\
\hline Bacillus mojavensis & 99.71 & 3 & 98.36 & 11 & 99.73 & 3 \\
\hline Bacillus subtilis spizizenii (ATCC 6633) & 99.65 & 3 & 98.02 & 13 & 99.6 & 3 \\
\hline B. subtilis spizizenii (DSM 15029) ${ }^{3}$ & 99.50 & 4 & 98.18 & 12 & 99.59 & 4 \\
\hline Bacillus atrophaeus & 99.23 & 5 & 97.63 & 15 & 99.39 & 5 \\
\hline Bacillus amyloliquefaciens & 99.07 & 7 & 97.43 & 16 & 99.26 & 7 \\
\hline Bacillus vallismortis & 99.05 & 8 & 97.52 & 16 & 99.19 & 8 \\
\hline Bacillus licheniformis & 97.41 & 15 & 98.62 & 12 & 97.18 & 15 \\
\hline Bacillus sonorensis & 96.86 & 17 & 98.50 & 12 & 96.59 & 17 \\
\hline Bacillus oleronius & 94.65 & 28 & 93.35 & 34 & 94.16 & 28 \\
\hline
\end{tabular}

${ }^{1}$ MicroSEQ bacterial library from Applied Biosystems (Foster City, CA; AB_Bacterial500Lib_2.0).

${ }^{2}$ ATCC $=$ American Type Culture Collection (Manassas, VA).

${ }^{3} \mathrm{DSM}=$ Deutsche Sammlung von Mikroorganismen und Zellkulturen (Braunschweig, Germany).

(with or without Sal), secretion direction, and their interaction in the model. All means are least squares means \pm SEM. Bars depicted in the figures generally represent the means of 12 observations. Technical difficulties prevented inclusion of the Sal invasion assay data from one run. Therefore, those means represent 9 observations. Comparisons of means were conducted only if a main effect or interaction was found to be significant $(P<0.05)$ in the model. Means were declared statistically different at $P<0.05$.

\section{RESULTS}

\section{Identification of Bacterial Specimens from Commercial Product}

The 3 different colonies recovered from the sample were sent to an accredited testing laboratory for microbiological and molecular (16S rRNA) analyses. The rRNA derived for the bacterial samples were matched with the library sequence database to find the closest phylogenetic neighbors. None of the genetic profiles showed complete similarity with the library database.
Specimens 1 (BS1) and 3 (BS3) had similarity scores with Bacillus subtilis subtilis (ATCC 6051) of 99.93 and $99.99 \%$, respectively (Table 1), and specimen 2 (BL2) was similar to BL at $98.1 \%$.

The precise alignments of each specimen, with its closest match, were then analyzed and the results are summarized in Table 2. The base differences that occur at the beginning ( 0 to 100 ) or at the end (400 to 500) of the sequence may be due to anomalies in the promoter attachment and have to be considered with caution. The differences observed in the interior of the sequence are more likely to be accurate. Both the BS1 and BS3 specimens exhibited 2 base differences compared with B. subtilis subtilis (ATCC 6051). In contrast, BL2 had a greater number of total mismatches (12) when compared with the BL from the library. However, when looking at the alignment, many of these mismatches occurred at the beginning and end of the sequence (base numbers $0,1,28,31,47,49,52$, and 439$)$.

All phylogenetic analyses clearly assigned the bacteria to the Bacillus genus. Specimens BS1 and BS3 were more precisely identified as part of the B. subtilis spp. and BL2 was identified as a member of the BL species.

Table 2. Location of mismatches of specimens obtained from a commercial product compared with reference sequences $^{1}$

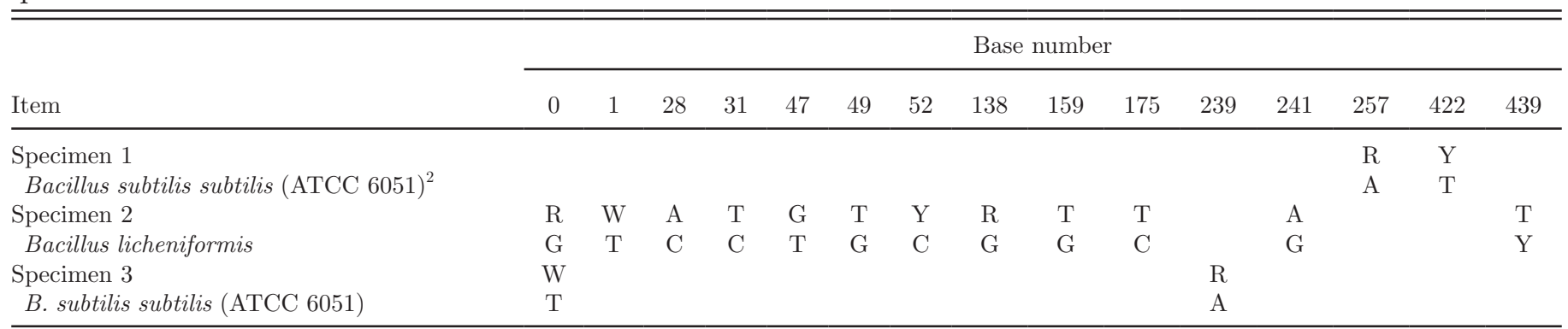

\footnotetext{
${ }^{1}$ The base number, in the top row, corresponds to the base position where a mismatch was observed. $\mathrm{A}=$ adenine; $\mathrm{T}=$ thymine; $\mathrm{G}=$ guanine; $\mathrm{C}=$ cytosine; $\mathrm{R}=\mathrm{A}$ or $\mathrm{G} ; \mathrm{W}=\mathrm{A}$ or $\mathrm{T} ; \mathrm{Y}=\mathrm{C}$ or $\mathrm{T}$.

${ }^{2} \mathrm{ATCC}=$ American Type Culture Collection (Manassas, VA).
} 
Trt $P<0.0001$

Dir $P<0.0001$

Trt $\times$ Dir $P<0.01$
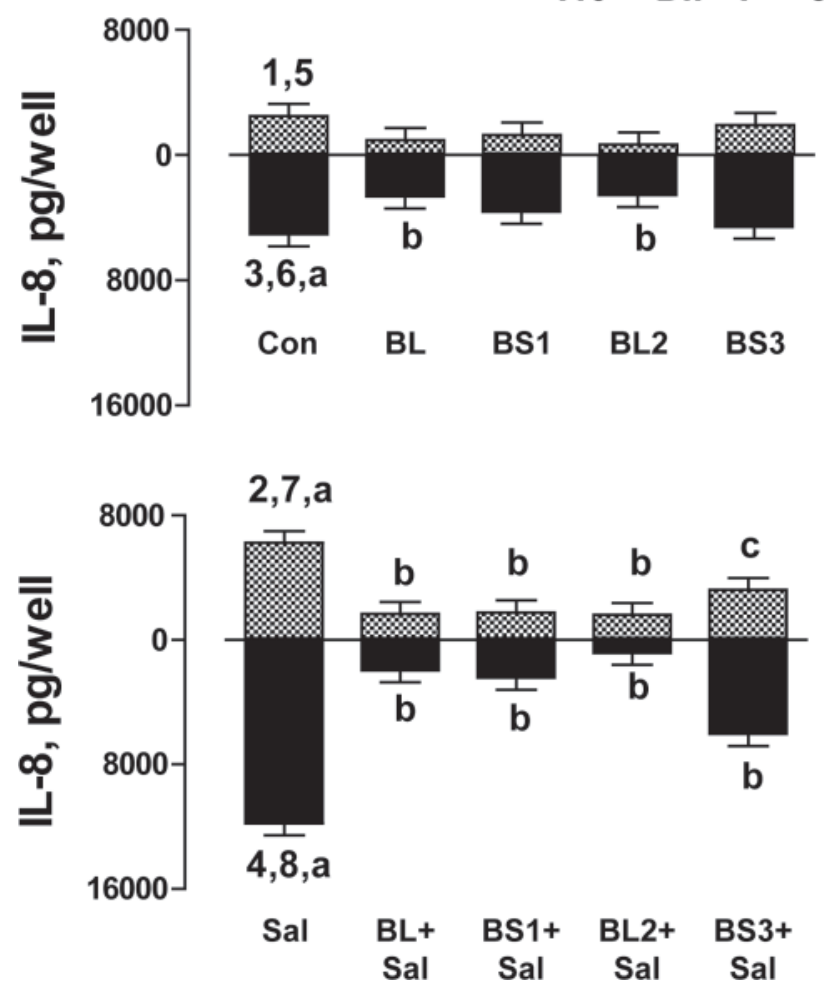

Figure 1. Polarized secretion of IL-8 from confluent porcine IPECJ2 intestinal epithelial cells. Secretion into the apical chamber is represented by the shaded bars, whereas secretion into the basolateral chamber is represented by the solid bars. Bars represent the mean \pm SEM of 12 replicate wells per treatment. Treatments included a control (Con) with media alone, or $17 \mathrm{~h}$ apical incubation with $108 \mathrm{cfu} /$ well of Bacillus licheniformis American Type Culture Collection strain (BL), Bacillus subtilis commercial isolate 1 (BS1), B. licheniformis commercial isolate 2 (BL2), or B. subtilis commercial isolate 3 (BS3; top panel). Additional treatments (bottom panel) included all Bacillus treatments exposed to $1 \mathrm{~h}$ of coculture with Salmonella enterica serovar Typhimurium (Sal) in the final hour of Bacillus incubation (BL+Sal, BS1+Sal, BL2+Sal, and BS3+Sal) or to S. enterica serovar Typhimurium for only $1 \mathrm{~h}$ (Sal). Media from the apical and basolateral compartments were removed and discarded. Cells were then washed, medium containing gentamicin was added, and the cells were returned to the incubator. After $4 \mathrm{~h}$, the experiment was terminated and media from the apical and basolateral compartments were removed for determination of IL-8. For analysis of the data, effects of treatment (Trt), secretion direction (Dir), and their interaction were included in the model. The numbers above and below the bars represent comparisons among interaction means between and within the Con and Sal treatments: 1 vs. 2 , apical Con vs. apical Sal, $P<0.001 ; 3$ vs. 4 , basolateral Con vs. basolateral Sal, $P<0.001 ; 5$ vs. 6 , apical Con vs. basolateral Con, $P<0.05 ; 7$ vs. 8 , apical Sal vs. basolateral Sal, $P<$ 0.001. Letters above and below the bars show comparisons between Con and other treatments (top panel) or between Sal and other treatments (bottom panel) within apical and basolateral means (a vs. b, $P$ $<0.001$; a vs. c, $P<0.01)$.

\section{$I L-8$}

In the absence of Bacillus coculture, exposure to Sal alone provoked an increase in IL- 8 secretion from IPECJ2 cells (Figure 1) compared with Con wells $(P<0.001$ for both the apical and basolateral directions). Both unstimulated secretion of IL-8, in Con wells, and stimulated secretion, in wells treated with Sal, were greater in the basolateral than in the apical direction $(P<$ 0.05 for control wells and $P<0.001$ for wells treated with Sal). Treatment with both BL isolates (BL and BL2) decreased basal secretion of IL-8 when compared with control wells $(P<0.05)$. Pretreatment with each Bacillus isolate followed by challenge with Sal reduced Sal-induced IL-8 secretion in both the apical and basolateral compartments compared with wells receiving only Sal $(P<0.001$; except for BS3 apical, $P<0.01)$.

\section{Effect of Bacillus Bacteria on Invasion of Sal into IPEC-J2 Cells}

Plate counts of Sal from lysates of cells preexposed to Bacillus spp. (BL+Sal, BS1+Sal, and BS3+Sal) were similar to plate counts from cells treated only with Sal (Figure 2). However, colonies of Sal that could be isolated from epithelial cell lysates in the BL2+Sal treatment were reduced compared with Sal alone $(P<0.001)$.

\section{Presence of Bacitracin in Media}

Our original intent was to evaluate bacitracin in media at the conclusion of the incubation after addition of Sal. Unfortunately, those media were discarded and only the media recovered at the conclusion of the study were evaluated for the presence of bacitracin. Bacitracin could be detected only in media from wells that had previously contained BL2 and BL2+Sal (Figure

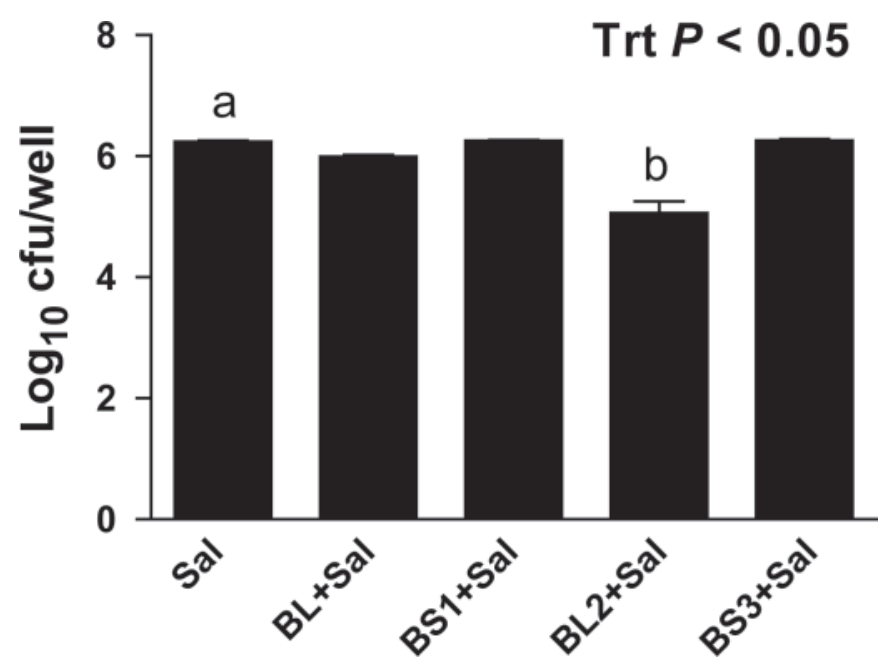

Figure 2. Invasion of Salmonella enterica serovar Typhimurium (Sal) into polarized confluent porcine IPEC-J2 intestinal epithelial cells. Bars represent the mean \pm SEM of 9 replicate wells per treatment. Treatments included apical $108 \mathrm{cfu}$ of Sal alone or 17 -h apical incubation with 108 cfu/well of Bacillus licheniformis American Type Culture Collection strain (BL), Bacillus subtilis commercial isolate 1 (BS1), B. licheniformis commercial isolate 2 (BL2), or B. subtilis commercial isolate 3 (BS3), and then coculture with Sal during the final hour of Bacillus incubation. Media from the apical and basolateral compartments were removed and discarded. Cells were then washed, medium containing gentamicin was added, and the cells were returned to the incubator. After $5 \mathrm{~h}$, the experiment was terminated. The IPECJ2 cells were lysed and the lysate was cultured overnight on tryptic soy agar for the presence of Sal. Colonies of Sal were reduced in BL2+Sal compared with Sal (a vs. b, $P<0.001$ ). Trt $=$ treatment. 
3). Concentrations of bacitracin were similar for BL2 and BL2+Sal in both the apical and the basolateral direction.

\section{Sal Sensitivity to Bacitracin}

Bacterial growth was observed in all the wells treated with Sal regardless of the dose of bacitracin tested. The microplate assay was qualitative, meaning that as long as bacterial growth resulted in a minimum predetermined level of absorbance, the level of bacitracin in the well failed to inhibit growth. It is possible that the greatest concentrations of bacitracin may have inhibited Sal growth, but that reduction would not have been detected based on the protocol we used.

\section{DISCUSSION}

Previous studies demonstrated that a common swine pathogen of the gastrointestinal tract, Sal, induced a proinflammatory response in the swine jejunal epithelial cell line IPEC-J2, as evidenced by secretion of the neutrophil chemoattractant IL-8 (Skjolaas et al., 2006). Basolaterally polarized secretion of IL- 8 has also been observed in epithelial cell lines from other species (McCormick et al., 1993; Vijay-Kumar et al., 2008). Of particular relevance to the current study, we previously reported that secretion of IL-8 from a model swine gastrointestinal epithelium was reduced substantially by pretreatment with the ATCC 10716 strain of BL (Skjolaas et al., 2007). Although a wide range of inflammatory mediators could have been evaluated in the current study, we evaluated elaboration of IL- 8 into the media owing to its well-documented role in attracting neutrophils to sites of mucosal inflammation.

In the current study, we sought to investigate Bacillus strains of more direct relevance to the swine industry to evaluate whether the antiinflammatory action observed with the laboratory strain could be extended to Bacillus bacteria found in direct-fed microbial preparations. For this, we turned to the feed additive BioPlus 2B. This additive is commercialized for use not only in pigs, but also in broilers and turkeys. It contains BL [Deutsche Sammlung von Microorganismen und Zellkulturen (DSM) 5749] spores isolated from soil and B. subtilis (DSM 5750) spores isolated from soybean fermentation. BioPlus 2B contains at least $1.6 \times 10^{9}$ spores/g of each Bacillus that are resistant to flavomycin and zinc-bacitracin. This product is reported to improve general health, fertility, and BW gain in swine production systems (Alexopoulos et al., 2004; Jørgensen and Kürti, 2006).

Our effort to obtain Bacillus bacteria from the product resulted in recovery of 3 isolates. The $16 \mathrm{~S}$ rRNA genetic analysis revealed the presence of $1 \mathrm{BL}(98.1 \%)$ and 2 B. subtilis (99.93 and $99.99 \%$ ). This is generally consistent with publically accessible information concerning the bacterial content of the product. However, the 2 B. subtilis we recovered (BS1 and BS3) differed
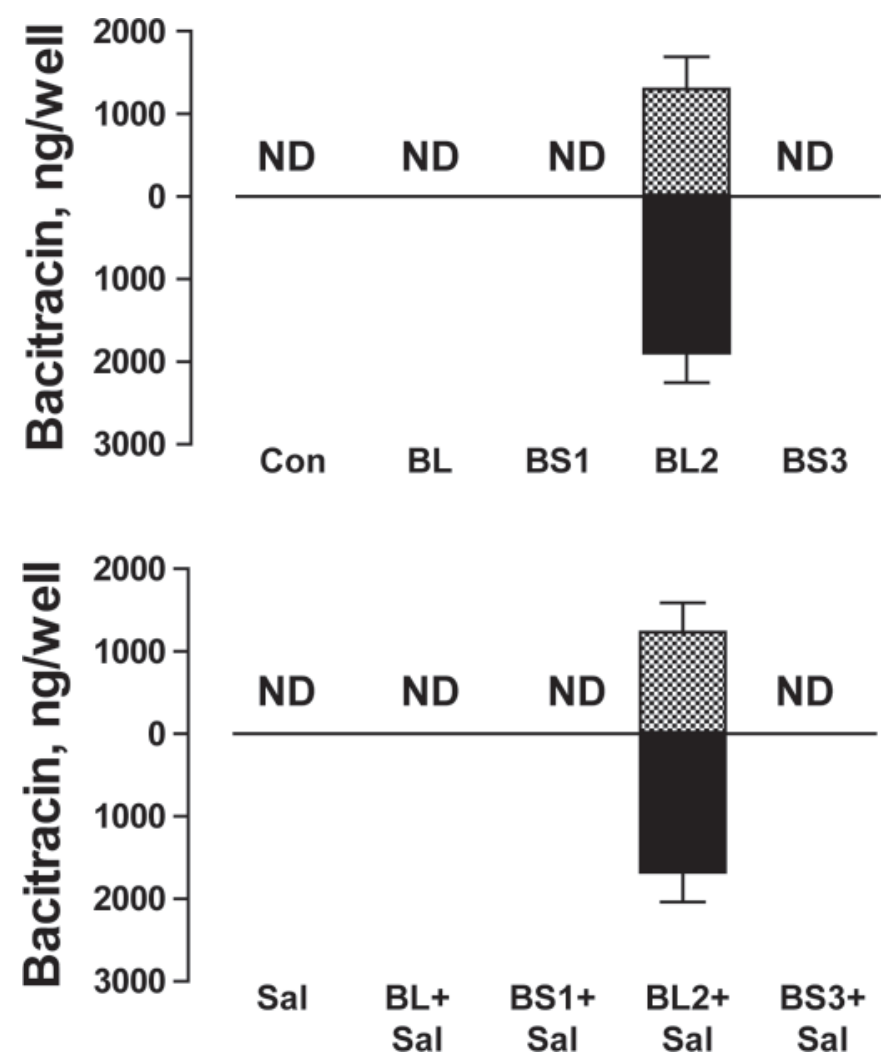

Figure 3. Concentration of bacitracin from confluent porcine IPEC-J2 intestinal epithelial cells. Accumulation in the apical chamber is represented by the shaded bars, whereas secretion into the basolateral chamber is represented by the solid bars. Bars represent the mean \pm SEM of 12 replicate wells per treatment. Treatments included a control (Con) with media alone or a 17-h apical incubation with $108 \mathrm{cfu} /$ well of Bacillus licheniformis American Type Culture Collection strain (BL), Bacillus subtilis commercial isolate 1 (BS1), B. licheniformis commercial isolate 2 (BL2), or B. subtilis commercial isolate 3 (BS3; top panel). Additional treatments (bottom panel) included all Bacillus treatments exposed to $1 \mathrm{~h}$ of coculture with Salmonella enterica serovar Typhimurium (Sal) in the final hour of Bacillus incubation (BL+Sal, BS1+Sal, BL2+Sal, and BS3+Sal) or to S. enterica serovar Typhimurium for only $1 \mathrm{~h}$ (Sal). Media from the apical and basolateral compartments were removed and discarded. Cells were then washed, medium containing gentamicin was added, and the cells were returned to the incubator. After $5 \mathrm{~h}$, the experiment was terminated and media from the apical and basolateral compartments were removed for determination of concentration of bacitracin. Bacitracin was not detectable (ND) in media from treatments other than BL2 and BL2+Sal.

by only 4 bases, and 2 of those bases were found at the extremity of the sequence. The information provided by the feed additive manufacturer indicated that the product contained equal amounts of spores from BL and B. subtilis. It could be that BS1 and BS3 are, in fact, the same B. subtilis, but given the slight difference underlined by the RNA analysis, we elected to evaluate the organisms separately.

In the current investigation, we again confirmed that the isolate of Sal that we have used in many in vivo (Balaji et al., 2000; Burkey et al., 2004; Fraser et al., 2007) and in vitro studies (Skjolaas et al., 2006, 2007) stimulated polarized secretion of IL-8 from IPEC-J2 cells. This effect has been thoroughly documented in this swine-derived cell line (Schierack et al., 2006) and in cell lines from other species (Eckmann et al., 1993; 
McCormick et al., 1993). Of relevance to the major focus of the current study, we again observed that the ATCC BL isolate completely inhibited Sal-induced secretion of IL-8 from IPEC-J2. Similarly, all isolates from the commercial feed additive behaved similarly to the ATCC BL isolate in blunting both apical and basolateral secretion of IL-8, although BS3 was somewhat less effective compared with the other strains. Of interest, both strains of BL (BL and BL2) even reduced basal IL-8 secretion from cells not stimulated with Sal. We had observed a similar effect previously, but only with Lactobacillus reuteri (Skjolaas et al., 2007).

One hypothesis to explain the ability of Bacillus bacteria to affect inflammatory signaling from enterocytes in vitro was that Bacillus prevented Sal from attaching and invading into the cell monolayer. To evaluate this possibility, we cultured lysates of IPEC-J2 after exposure to Bacillus. When compared with Sal, only BL2+Sal reduced colonies of Sal that could be recultured out of IPEC-J2 lysates. Although graphically, the reduction does not appear to be substantial on a logarithmic scale, it likely indicates a marked decline in the number of Sal breaching the epithelial barrier in the BL2+Sal treatment. Although this reduction may be related to other factors (discussed below), it does not explain the general ability of Bacillus to reduce secretion of IL-8 under these experimental circumstances because BL, BS1, and BS3 all reduced IL-8 without affecting invasion.

Among other secondary secretory components, Bacillus bacteria, including the ATCC BL isolate, are known to produce the polypeptide antibiotic bacitracin (Konz et al., 1997). We hypothesized that the ability of $\mathrm{Ba}$ cillus bacteria, particularly BL, to affect Sal invading IPEC-J2 cells may simply be related to their ability to produce bacitracin. As noted previously, our intent was to measure bacitracin in media collected at the conclusion of the bacterial incubation. Unfortunately, those media were discarded and only media collected at the conclusion of the study were available for determination of bacitracin content.

Bacitracin was detected only in media from wells that had contained BL2, both in the presence and absence of Sal. In our antibiotic sensitivity tests, bacitracin failed to prevent Sal growth at concentrations up to $20 \mu \mathrm{g} / \mathrm{mL}$. Because we did not measure bacitracin at the conclusion of the bacterial incubation, we cannot say with absolute certainty that other BL2 or other bacilli produced bacitracin at quantities sufficient to affect the viability of Sal in our cultures. Thus, we must limit our interpretation and discussion here to account for the residual presence of bacitracin in the absence of Bacillus organisms and after thorough washing of wells. Key to our interpretation, we noted the gross appearance of biofilm associated with cultures of BL2 as we gained early experience growing the isolate. Bacillus bacteria are well-known producers of a variety of metabolites, including surfactin. Bacillus subtilis, for example, is known to produce a large array of secondary metabolites, such as mycosubtilin, iturin, and surfactin (Arima et al., 1968; Stein, 2005; Seydlova and Svobodova, 2008). Surfactin is a lipopeptide antibiotic and a powerful biosurfactant (Singh and Cameotra, 2004; Rodrigues et al., 2006; Nagorska et al., 2007). Surfactin, among its many properties, exhibits antimicrobial activities. Therefore, we suggest that BL2-produced biofilm that was resistant to washing after bacterial incubation could likely have been the source of passive evolution of bacitracin in the media in the absence of a direct source from Bacillus bacteria. Whether sufficient bacitracin was produced to affect survival of Sal in the BL2+S treatment cannot be determined from the current data.

Finally, we feel it is important to consider whether the effects of Bacillus on Sal-induced IL- 8 secretion we have observed here and previously (Skjolaas et al., 2007) have physiological relevance. Perhaps it could be that these effects might simply be an artifactual consequence of the pretreatment with Bacillus bacteria exhausting the nutrients in the media, leaving the cells less capable of secreting IL-8. On one hand, the production of bacitracin is generally associated with the early stages of sporulation in BL (Bernlohr and Novelli, 1959), and this, coupled with the obvious acidity (yellowing) that developed in the media by the time Sal were added, might support such a conclusion. On the other hand, under identical experimental conditions, a lactic acidproducing bacteria, L. reuteri, produced substantial acidity in the media, but this condition alone failed to reduce the ability of Sal to stimulate IL-8 secretion from IPEC-J2 (Skjolaas et al., 2007). Assuming our findings have relevance to the function of Bacillus-containing feed additives within the gastrointestinal tract, an important question that remains relates to the ability of these bacteria, or any direct-fed microbial, to colonize the gut in sufficient numbers to affect the interaction of the epithelium with enteropathogens to explain the reported benefits of probiotic bacteria.

Results of the current studies indicate that Bacillus bacteria, at least B. subtilis and BL, have the ability to intervene in secretion of the neutrophil chemoattractant IL-8 from swine intestinal epithelial cells. This effect on chemokine secretion by gastrointestinal epithelial cells in vitro could not be explained by reduced invasion of epithelial cells by Sal.

\section{LITERATURE CITED}

Alexopoulos, C., I. E. Georgoulakis, A. Tzivara, C. S. Kyriakis, A. Govaris, and S. C. Kyriakis. 2004. Field evaluation of the effect of a probiotic-containing Bacillus licheniformis and Bacillus subtilis spores on the health status, performance, and carcass quality of grower and finisher pigs. J. Vet. Med. A, Physiol. Pathol. Clin. Med. 51:306-312.

Arima, K., A. Kakinuma, and G. Tamura. 1968. Surfactin, a crystalline peptidelipid surfactant produced by Bacillus subtilis: Isolation, characterization and its inhibition of fibrin clot formation. Biochem. Biophys. Res. Commun. 31:488-494.

Balaji, R., K. J. Wright, C. M. Hill, S. S. Dritz, E. L. Knoppel, and J. E. Minton. 2000. Acute phase responses of pigs challenged 
orally with Salmonella typhimurium. J. Anim. Sci. 78:18851891.

Bernlohr, R. W., and G. D. Novelli. 1959. Antibiotic production as a function of spore formation in Bacillus licheniformis. Nature 184:1256-1257.

Burkey, T. E., K. A. Skjolaas-Wilson, and J. E. Minton. 2004. Time course of polarized interleukin-8 (IL-8) secretion in swine jejunal epithelial cells following apical lipopolysaccharide (LPS) and Salmonella enterica serovar Typhimurium (ST) or Choleraesuis (SC). FASEB J. 18:A818-A819.

Dritz, S. S., M. D. Tokach, R. D. Goodband, and J. L. Nelssen. 2002. Effects of administration of antimicrobials in feed on growth rate and feed efficiency of pigs in multisite production systems. J. Am. Vet. Med. Assoc. 220:1690-1695.

Eckmann, L., M. F. Kagnoff, and J. Fierer. 1993. Epithelial-cells secrete the chemokine interleukin- 8 in response to bacterial entry. Infect. Immun. 61:4569-4574.

Fraser, J. N., B. L. Davis, K. A. Skjolaas, T. E. Burkey, S. S. Dritz, B. J. Johnson, and J. E. Minton. 2007. Effects of feeding Salmonella enterica serovar Typhimurium or serovar Choleraesuis on growth performance and circulating insulin-like growth factor- 1 , tumor necrosis factor- $\alpha$, and interleukin- $1 \beta$ in weaned pigs. J. Anim. Sci. 85:1161-1167.

Godsey, B. H., K. A. Skjolaas, and J. E. Minton. 2007. Pre-exposure to Bacillus licheniformis reduces interleukin-8 response of swine intestinal epithelial cells to Salmonella enterica serovar Typhimurium. J. Anim. Sci. 85:146-147. (Abstr.)

Jørgensen, J. N., and P. Kürti. 2006. Novel approach to reduce preweaning mortality. Int. Pig Top. 19:11-13.

Konz, D., A. Klens, K. Schorgendorfer, and M. A. Marahiel. 1997. The bacitracin biosynthesis operon of Bacillus licheniformis ATCC 10716: Molecular characterization of three multi-modular peptide synthetases. Chem. Biol. 4:927-937.

McCormick, B. A., S. P. Colgan, C. Delp-Archer, S. I. Miller, and J. L. Madara. 1993. Salmonella typhimurium attachment to human intestinal epithelial monolayers: Transcellular signaling to subepithelial neutrophils. J. Cell Biol. 123:895-907.

Nagórska, K., M. Bikowski, and M. Obuchowskji. 2007. Multicellular behaviour and production of a wide variety of toxic substances support usage of Bacillus subtilis as a powerful biocontrol agent. Acta Biochim. Pol. 54:495-508.

Rhoads, J. M., W. Chen, P. Chu, H. M. Berschneider, R. A. Argenzio, and A. M. Paradiso. 1994. L-Glutamine and L-asparagine stimulate $\mathrm{Na}^{+}-\mathrm{H}^{+}$exchange in porcine jejunal enterocytes. Am. J. Physiol. 266:G828-G838.

Rodrigues, L., I. M. Banat, J. Teixeira, and R. Oliveira. 2006. Biosurfactants: Potential applications in medicine. J. Antimicrob. Chemother. 57:609-618.

Schierack, P., M. Nordhoff, M. Pollmann, K. D. Weyrauch, S. Amasheh, U. Lodemann, J. Jores, B. Tachu, S. Kleta, A. Blikslager, K. Tedin, and L. H. Wieler. 2006. Characterization of a porcine intestinal epithelial cell line for in vitro studies of microbial pathogenesis in swine. Histochem. Cell Biol. 125:293305.

Seydlova, G., and J. Svobodova. 2008. Review of surfactin chemical properties and the potential biomedical applications. Cent. Eur. J. Med. 3:123-133.

Singh, P., and S. S. Cameotra. 2004. Potential applications of microbial surfactants in biomedical sciences. Trends Biotechnol. $22: 142-146$.

Skjolaas, K. A., T. E. Burkey, S. S. Dritz, and J. E. Minton. 2006. Effects of Salmonella enterica serovars Typhimurium (ST) and Choleraesuis (SC) on chemokine and cytokine expression in swine ileum and jejunal epithelial cells. Vet. Immunol. Immunopathol. 111:199-209.

Skjolaas, K. A., T. E. Burkey, S. S. Dritz, and J. E. Minton. 2007. Effects of Salmonella enterica serovar Typhimurium, or serovar Choleraesuis, Lactobacillus reuteri and Bacillus licheniformis on chemokine and cytokine expression in the swine jejunal epithelial cell line, IPEC-J2. Vet. Immunol. Immunopathol. 115:299-308

Stein, T. 2005. Bacillus subtilis antibiotics: Structures, syntheses and specific functions. Mol. Microbiol. 56:845-857.

US Environmental Protection Agency. 1997a. Bacillus licheniformis final risk assessment, Biotechnology Program Under Toxic Substances Control Act. US Environ. Prot. Agency, Washington, DC.

US Environmental Protection Agency. 1997b. Bacillus subtilis final risk assessment, Biotechnology Program Under Toxic Substances Control Act. US Environ. Prot. Agency, Washington, DC.

Vijay-Kumar, M., J. D. Aitken, and A. T. Gewirtz. 2008. Toll like receptor-5: Protecting the gut from enteric microbes. Semin. Immunopathol. 30:11-21. 